\title{
Impaired colonization of the gonads by primordial germ cells in mice lacking a chemokine, stromal cell-derived factor-1 (SDF-1)
}

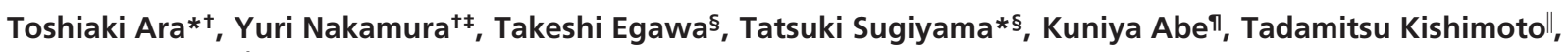 \\ Yasuhisa Matsui ${ }^{\ddagger}$, and Takashi Nagasawa*,** \\ *Department of Medical Systems Control, Institute for Frontier Medical Sciences, Kyoto University, 53 Kawahara-cho, Shogoin, Sakyo-ku, Kyoto 606-8507, \\ Japan; Departments of ₹Molecular Embryology and §Immunology, Research Institute, Osaka Medical Center for Maternal and Child Health, \\ 840 Murodo-cho, Izumi, Osaka 594-1101, Japan; "Technology Development Team for Mammalian Cellular Dynamics, The Institute of \\ Physical and Chemical Research BioSource Center, 3-1-1 Kouyadai, Tsukuba, Ibaraki 305-0074, Japan; and IIOsaka University, \\ 1-1 Yamadaoka, Suita, Osaka 565-0871, Japan
}

Contributed by Tadamitsu Kishimoto, February 6, 2003

Primordial germ cells (PGCs) are the founders of sperm or oocytes. PGCs migrate through the tissues of the embryos and colonize the gonads during development. However, the cytokines essential for colonization of the gonads by PGCs in mammals remain unclear. Stromal cell-derived factor-1 (SDF-1, also called PBSF and CXCL12) is a member of chemokines, a family of structurally related chemoattractive cytokines. SDF-1 and its primary physiologic receptor CXCR4 have multiple essential functions in development including colonization of bone marrow by hematopoietic cells and neuron localization within cerebellum during embryogenesis as well as $B$ lymphopoiesis and cardiovasculogenesis. Here, we have shown that PGCs have cell-surface expression of CXCR4 and that, in SDF-1 ${ }^{-/-}$mice, PGCs undergo directed migration through tissues of embryos, but the numbers of PGCs in the gonads are significantly reduced. The proliferation of PGCs within the gonads seems normal in the mutant mice. These findings reveal the essential role for SDF-1 in murine PGC development likely by controlling colonization of the gonads by PGCs.

$\mathbf{S}_{\mathrm{n}}^{\mathrm{t}}$ tem cells migrate, colonize, and proliferate during development. Primordial germ cells (PGCs) are the founders of sperm or oocytes. PGCs arise in the root of the developing allantois, then enter into the hindgut endoderm, migrate through the mesentery of hindgut, and colonize the genital ridges in the mouse (1). Cytokines play an important role in regulating these processes. The experiments using mutant mice with targeted gene disruption have revealed the essential roles of several cytokines in PGC development. Transforming growth factor $\beta$ superfamily proteins have been shown to be critical for the development of PGCs. Mice lacking bone morphogenetic protein-4 (BMP-4) contain no PGCs (2), and mice lacking another transforming growth factor- $\beta$ family member, BMP- $8 \mathrm{~b}$, have severely reduced numbers of PGCs (3), indicating that the initiation of PGC has been shown to depend on BMP-4 and -8 . On the other hand, $\mathrm{Sl} / \mathrm{Sl}$ or $\mathrm{W} / \mathrm{W}$ mutant mice that lack the activities of stem cell factor or its receptor c-kit lack PGCs, and stem cell factor promotes the survival of PGCs in vitro, indicating that stem cell factor is essential for PGC survival during development (4-7). Analysis using We/We mice has suggested that c-kit is involved in migration of PGCs through the mesentery of hindgut (8). Despite these studies, the cytokines that are essential for colonization of the genital ridges by PGCs remain unclear.

Stromal cell-derived factor-1/pre-B cell growth-stimulating factor/CXC chemokine ligand 12 (SDF-1/PBSF/CXCL12) is a member of chemokines, a large family of structurally related chemoattractive cytokines, which act via a seven-transmembrane spanning G protein-coupled receptor (9). SDF-1 was isolated from bone marrow stromal cells and first characterized as a pre-B cell growth-stimulating factor $(10,11)$. CXCR4 has been shown to be a primary physiologic receptor for SDF-1 (12-18) and also function as an entry coreceptor for strains of HIV-1 (19). Studies using mutant mice with targeted gene disruption have revealed that SDF-1 and CXCR4 are essential for $\mathrm{B}$ lymphocyte development, blood vessel formation in gastrointestinal tract, cardiac ventricular septum formation, neuron localization within cerebellum, and embryonic viability $(15-18,20)$. Of particular note, SDF-1 $1^{-/-}$or $\mathrm{CXCR} 4^{-/-}$embryos have much more impaired myelopoiesis in bone marrow compared with myelopoiesis in fetal liver, suggesting that SDF-1 and CXCR4 are involved in colonization of bone marrow by hematopoietic cells during embryogenesis $(11,15-18)$. Consistent with this, hematopoietic progenitors migrate in response to SDF-1 in vitro $(21,22)$. These results prompt us to study the involvement of SDF-1 in colonization of the gonad by PGCs during development in which there is a dynamic movement of PGCs, using SDF-1-1or $\mathrm{CXCR} 4^{-1-}$ mice. Here, we show that SDF-1 is not required for directed migration through tissues of embryos but instead is essential for the homing of PGCs into the genital ridges. Our results reveal the essential role for SDF-1 in the development of PGCs in mammals.

\section{Materials and Methods}

Mice. The generation of SDF-1 $1^{-/-}$or $\mathrm{CXCR} 4^{-/-}$mice has been described $(15,16)$. Heterozygotes were backcrossed more than 10 times with C57BL/6 mice. Oct-3/4 GFP transgenic mice have been described (23).

To generate the mice in which GFP gene was knocked into the SDF-1 locus (SDF-1/GFP knock-in mice), exon 2 of the SDF-1 gene was replaced by GFP expression cassette by homologous recombination in embryonic stem cells (15). Mutated embryonic stem colonies were used to produce mice hemizygous for the GFP insertion by blastocyst injection as described (15). Mice hemizygous for the GFP insertion that have one functional SDF-1 allele are phenotypically normal and can be used for the analysis of SDF-1 expression.

Detecting and Counting PGCs. Alkaline phosphatase staining was performed as described (2). Anti-4C9 antibody (a kind gift of T. Muramatsu, Nagoya University, Nagoya, Japan; ref. 24) was used to detect PGCs on Bouin-fixed paraffin sections (5 $\mu \mathrm{m}$ thick). Sections were visualized using Vectastain ABC kit (Vector Laboratories) and diaminobenzidine-peroxidase solution. $4 \mathrm{C}^{+}$ cells were counted on each section.

Abbreviations: SDF-1, stromal cell-derived factor-1; PGC, primordial germ cell; BMP, bone morphogenetic protein; En, embryonic day $n$.

${ }^{\dagger}$ T.A. and Y.N. contributed equally to this work.

**To whom correspondence should be addressed. E-mail: tnagasa@frontier.kyoto-u.ac.jp. 
BrdUrd Labeling, Immunohistochemical Staining, and Confocal Microscopy. Pregnant mice were injected intravenously with BrdUrd $(100 \mathrm{mg} / \mathrm{kg}$ of body weight $)$ in saline. After $2 \mathrm{~h}$, tissues were frozen in OCT compound (Tissue-Tek, Sakura Finetechnical, Tokyo) and cut into $10-\mu \mathrm{m}$-thick sections. After fixation in $4 \%$ paraformaldehyde, sections were treated with $2 \mathrm{M} \mathrm{HCl}$ for 20 min at room temperature and permeabilized in PBS containing $0.3 \%$ Triton $\mathrm{X}-100$. Sections were incubated with anti-4C9 and anti-BrdUrd antibodies (Dako) followed by FITC-conjugated anti-rat IgM (Cappel) and Alexa546-conjugated anti-mouse IgG (Molecular Probes). Then, sections were mounted with the Slowfade Antifade kit (Molecular Probes).

For detection of GFP, tissues from GFP/SDF-1 knock-in embryos were dissected, fixed in $4 \%$ paraformaldehyde for overnight at $4{ }^{\circ} \mathrm{C}$, and directly observed. All confocal microscopy was carried out on an LSM 5 PASCAL (Zeiss).

Flow Cytometry Analysis. Male Oct-3/4 GFP transgenic mice were mated with female mice of ICR. Genital ridges were incubated with collagenase (type I, Sigma; final concentration $0.12 \%$ $\mathrm{vol} / \mathrm{vol}$ ) for $1 \mathrm{~h}$ at $37^{\circ} \mathrm{C}$ in PBS containing $10 \% \mathrm{FCS}$. Cells were dispersed, washed, and resuspended in DMEM containing $2 \%$ FCS. Cells were stained with biotinylated anti-CXCR4 (2B11) or isotype control $\mathrm{IgG} 2 \mathrm{~b} \kappa$ (A95-1) followed by allophycocyaninconjugated streptavidin. All antibodies and reagents were purchased from PharMingen. Flow cytometry was performed with FACSCalibur and analyzed with CELLQUEST software (Becton Dickinson).

RT-PCR. Total RNA was isolated from sorted $\mathrm{GFP}^{+}$PGCs in Oct-3/4 GFP transgenic mice, treated with DNase I, and reverse-transcribed. cDNA was amplified by PCR using genespecific primers. PCR primers are $5^{\prime}$-taggatcttcctgcccaccat- $3^{\prime}$ (sense) and 5' -tgaccaggatcaccaatcca-3' (antisense) for CXCR4 and $5^{\prime}$-cctgctggattacattaaagcactg-3' and $5^{\prime}$-gtcaagggcatatccaacaacaaac-3' for hypoxanthine-guanine phosphoribosyltransferase. The PCR products were analyzed with an Agilent 2100 Bioanalyzer or separated by agarose gel electrophoresis.

\section{Results}

Migration of PGCs Through Tissues of Embryos Occurs in SDF-1 ${ }^{-1-}$ Mice. In the mouse embryos, PGCs are first seen in the root of the extraembryonic mesoderm at embryonic day 7.5 (E7.5), enter into the hindgut endoderm at E8, migrate through the mesentery of hindgut from E9.5 to E10.5, and colonize the genital ridges around E10.5. To examine the physiological role of a chemokine SDF-1 in the development of PGCs, we measured the numbers of PGCs along development in SDF-1 ${ }^{-1-}$ embryos. Because PGCs have expression of tissue nonspecific alkaline phosphatase $(25,26)$, alkaline phosphatase staining analysis was used to detect PGCs in whole-mount mouse embryos. This analysis revealed that the numbers of PGCs in the hindgut endoderm and mesentery were unaffected or slightly reduced in SDF-1 $1^{-/-}$embryos at E9.5 compared with control embryos, indicating that SDF-1 was not required for generation of PGCs, emigration of PGCs into the migration route, or directed migration of PGCs through tissues of embryos (Fig. 1). Thereafter, PGCs emigrate into the genital ridges, and $>40 \%$ of PGCs were found in genital ridges in control embryos at E10.5. However, only $14 \%$ of PGCs were observed in the genital ridges from SDF-1 $1^{-1-}$ embryos (data not shown). The numbers of PGCs that remained in hindgut endoderm or mesentery were larger in the mutants compared with control embryos (Fig. $2 \mathrm{~A}$ and $B$ ). At E11.5, although almost all of PGCs were found in the genital ridges in control embryos, the numbers of PGCs in the genital ridges were reduced in $\mathrm{SDF}-1^{-1-}$ embryos, and a small population of PGCs was still located in hindgut endoderm or mesentery (Fig. 2A). Despite altered PGC distribution, ectopic

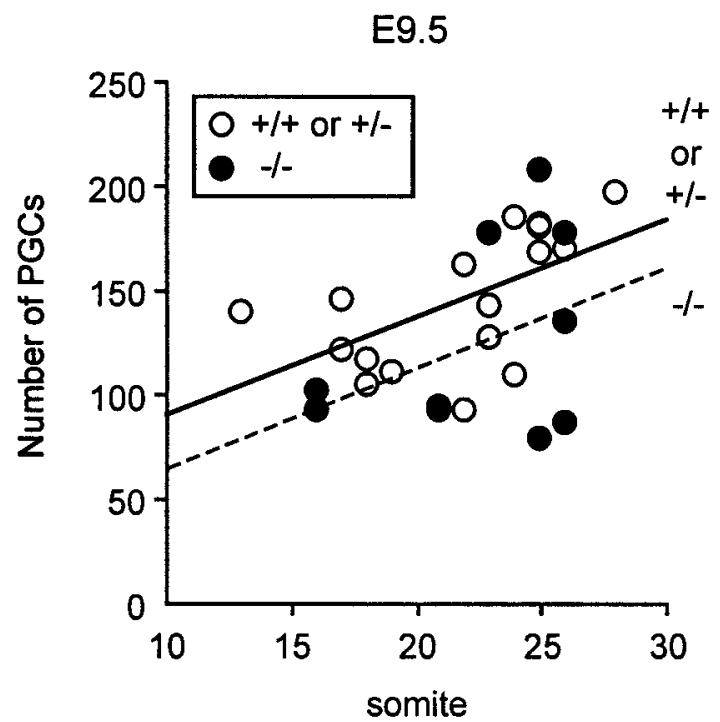

Fig. 1. Relatively normal numbers of PGCs in the route of migration in SDF- $1^{-1-}$ embryos at E9.5. Linear regression analysis of PGC numbers (counted in whole-mount) vs. somite numbers in control and SDF-1-1- embryos at E9.5.

PGCs located outside the migration route were not observed in SDF-1 ${ }^{-1-}$ embryos at E9.5, E10.5, or E11.5. Consistent with this, the total numbers of PGCs within the migration route and genital ridges in SDF-1 $1^{-/-}$embryos were comparable to control embryos at E10.5 as well as E9.5 (Figs. 1 and 2C). Together, it is likely that PGCs migrate through tissues of embryos, but the migration was delayed in SDF-1 $1^{-1-}$ embryos.

Colonization of the Gonads by PGCs Is Impaired in SDF-1 ${ }^{-1-}$ Mice. At E12.5, PGCs that remained in the mesentery of hindgut were not observed in both control and SDF-1 $1^{-/-}$embryos in the wholemount alkaline phosphatase staining analysis, suggesting that almost all PGCs colonized the gonads in these mice. Alkaline phosphatase staining analysis or immunohistochemistry with PGC-specific marker 4C9 (24) on paraffin sections revealed that the numbers of PGCs were significantly reduced in the gonads from SDF-1 $1^{-1-}$ embryos compared with control embryos at E12.5 (data not shown; Fig. 2D). Then, we analyzed the proliferation of PGCs in the gonads by BrdUrd labeling of whole embryos at E12.5. A small population of BrdUrd-labeled cells could be identified in both control and SDF-1 ${ }^{-1-}$ embryos, suggesting that reduction in the numbers of PGCs in SDF-1-/embryos was not due to impaired proliferation of PGCs in the gonads (Fig. 2E). After colonization of the gonads, PGCs actively proliferate, and the numbers of PGCs increase to a maximum at around E13.5 or E14.5 when the male PGCs enter mitotic arrest and the female PGCs enter meiosis. Immunohistochemistry with 4C9 on paraffin sections at E13.5 or E14.5 revealed that the numbers of PGCs in gonads from SDF-1 ${ }^{-1-}$ embryos were significantly smaller than those in control embryos (Fig. $2 D$ and $F$ ). However, the increase of PGCs within the gonads from E12.5 to E13.5 was similar in control and SDF-1 ${ }^{-/-}$ embryos (male, 2.8-fold versus 2.3-fold; female, 1.9-fold versus 2.1 -fold, respectively), suggesting that the reduction of PGCs in the SDF-1 $1^{-1-}$ gonads was not due to the impaired expansion of PGCs in the gonads but was instead due to the impaired emigration of PGCs into the gonads (Fig. 2D). PGCs aggregate with each other in the wild-type gonads, and these PGC aggregates were also observed in SDF-1 $1^{-1-}$ gonads (Fig. $2 F$ ). These defects in PGC development were observed when we analyzed the mice lacking CXCR4, a primary physiologic receptor for SDF-1 (data not shown). 

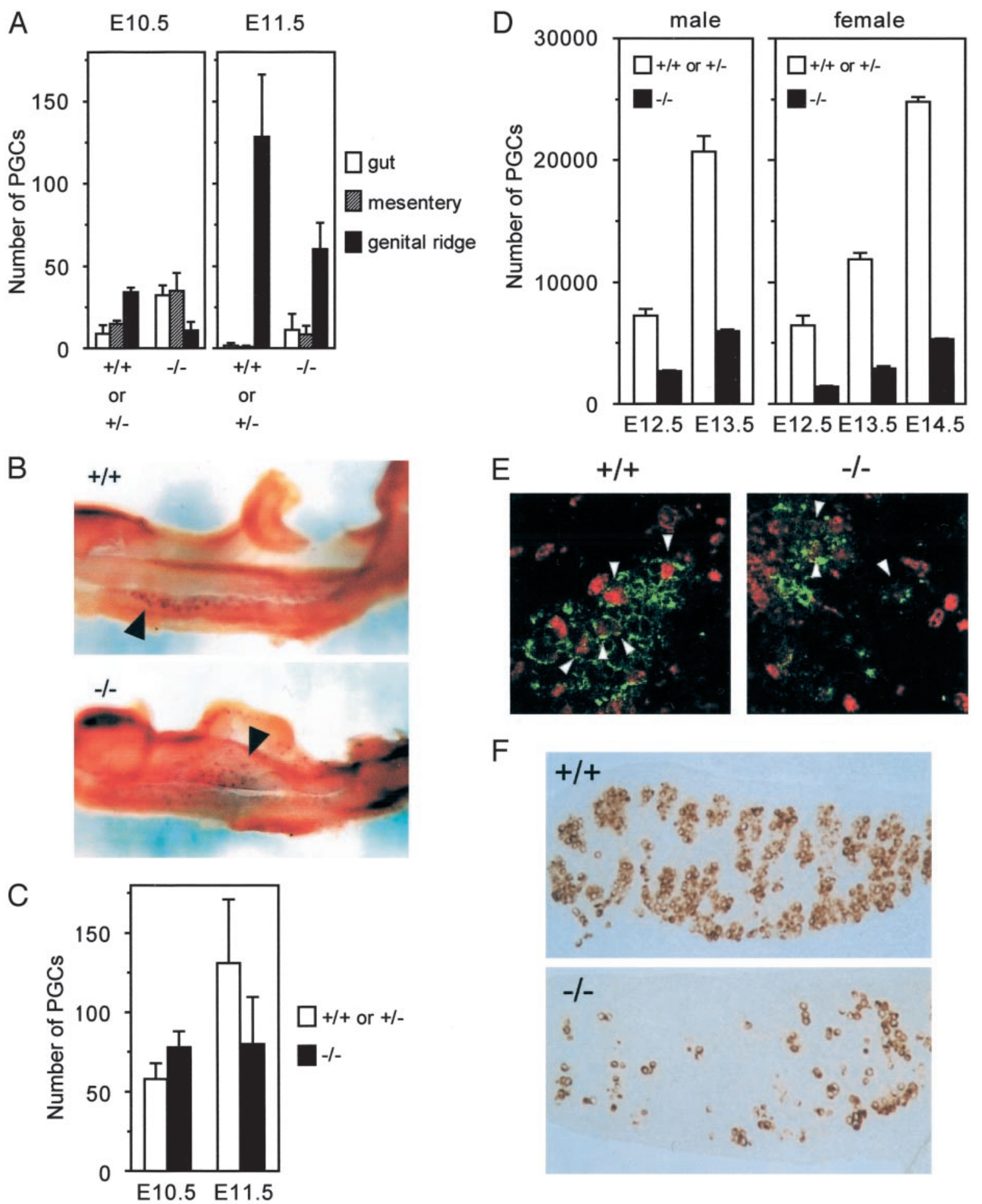

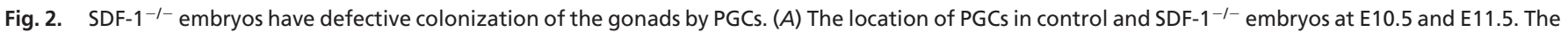

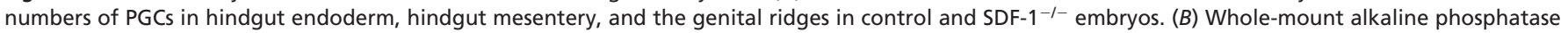

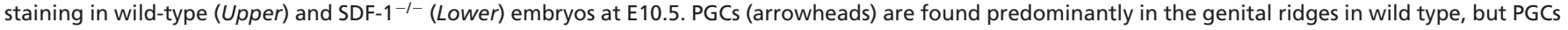

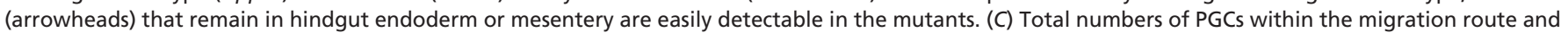

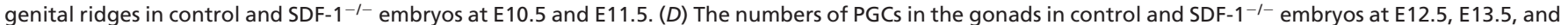

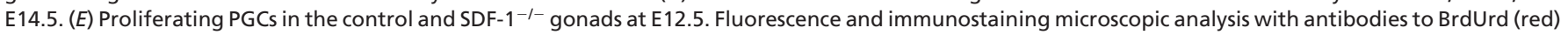

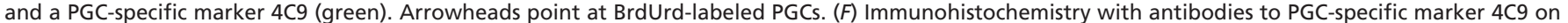
paraffin sections of the male E13.5 gonads from control and SDF-1 $1^{-1-}$ embryos.

It has been shown that SDF-1 and CXCR4 are essential for organ vascularization (16). SDF-1 $1^{-1-}$ mice have defective formation of large vessels supplying the gastrointestinal tract (16).
Whole-mount immunohistological analysis using PECAM-1, a cell-adhesion molecule expressed in endothelium, revealed that there was no significant difference in vasculature of E12.5 


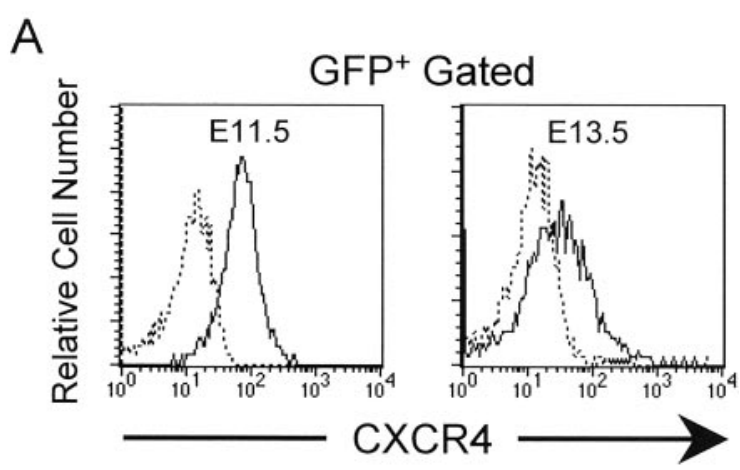

B

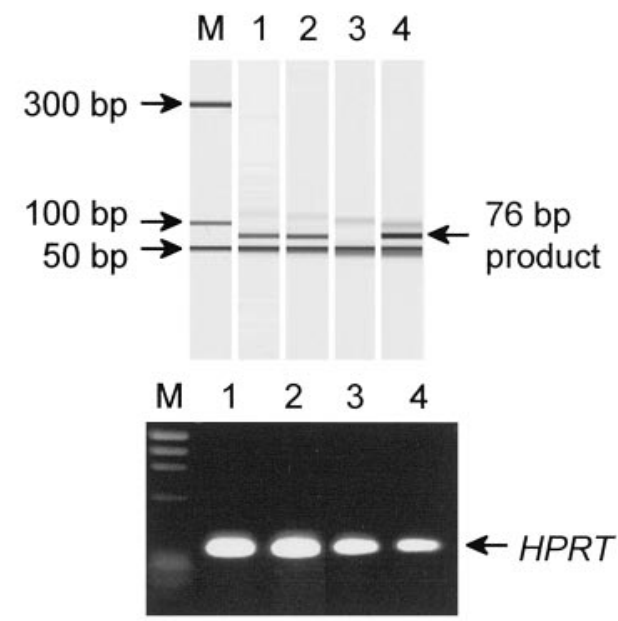

C

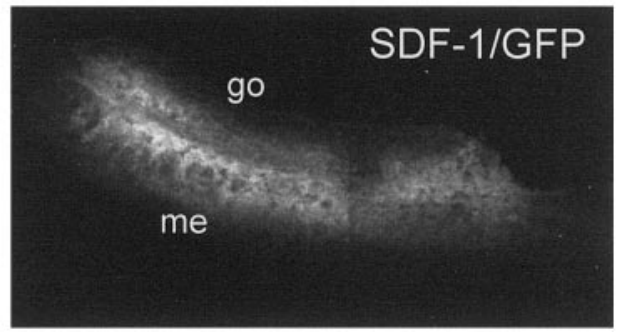

Fig. 3. Expression of CXCR4 and SDF-1 in murine PGCS or the developing genital ridge. (A) Flow cytometric analysis of cell-surface expression of CXCR4 on PGCs at E11.5 and E13.5, using transgenic mice expressing GFP in PGCs. $\mathrm{GFP}^{+} \mathrm{PGCs}$ have significant cell-surface expression of CXCR4. Solid lines represent staining with CXCR4. Dashed lines represent background staining with isotype-matched controls. ( $B$ ) RT-PCR analysis of CXCR4 gene expression with E11.5 or E13.5 PGCs. M, molecular marker; lane 1, E11.5 GFP ${ }^{+}$PGCs; lane 2, E13.5 male GFP ${ }^{+}$PGCs; lane 3, E11.5 GFP ${ }^{-}$cells; lane 4, pre-B cell clone, DW34 (10). The CXCR4 RT-PCR products of $76 \mathrm{bp}$ are identified. (C) The expression pattern of SDF-1 in the E12.5 gonad (go) and subjacent mesonephric tissue (me) from the embryo in which GFP gene is knocked into the SDF-1 locus.

gonads between control and SDF-1 $1^{-/-}$embryos, suggesting that defects in PGC development were not due to the deficits in blood vessel formation in the gonads (data not shown).

Expression of SDF-1 or CXCR4 in the Developing Genital Ridge or PGCs. Subsequently, we examined the expression of CXCR4 in PGCs. Two approaches with flow cytometry and RT-PCR were used. First, we analyzed the cell-surface expression of CXCR4 protein on live PGCs by using transgenic mice expressing GFP in PGCs under the control of the Oct-3/4 gene regulatory sequences (Oct-3/4 GFP transgenic mice; ref. 23). Flow cytometric analysis revealed that all $\mathrm{GFP}^{+}$PGCs expressed a high level of CXCR4 at E11.5, when PGCs migrated and entered the gonads (Fig. $3 A$ ). In contrast, the expression of CXCR4 was down-regulated at E13.5, when PGCs were no longer motile (Fig. $3 A$ ). Second, we sorted $\mathrm{GFP}^{+}$PGCs from genital ridges in Oct-3/4 GFP transgenic mice and performed RT-PCR with CXCR4-specific primers. Consistent with the data obtained by flow cytometry, we found the expression of CXCR4 mRNA in $\mathrm{GFP}^{+} \mathrm{PGC}$ fractions at E11.5 and E13.5 (Fig. 3B). We next examined the expression pattern of SDF-1 in the embryos by using mice in which GFP gene was knocked into the SDF-1 locus (SDF-1/GFP knock-in mice). Expression of SDF-1 was observed in the root of the gonad and mesonephric tissue adjacent to the gonad at E12.5 (Fig. 3C). These expression patterns of CXCR4 and SDF-1 suggest that SDF-1 acts directly on PGCs and regulates their emigration into the gonads through the neighboring tissues.

\section{Discussion}

PGC development consists of several processes including initiation, survival, proliferation, migration, and homing of PGCs. First, because PGCs migrate through the tissues of embryos but the migration was delayed in SDF-1 $1^{-1-}$ embryos, it is likely that SDF-1 is not required for directing PGCs through migration route; instead, it plays a role in promoting the migration of PGCs. Second, the results that the numbers of PGCs in the gonads were significantly reduced in SDF-1-1embryos but that their increase within the gonads was similar in control and SDF-1 $1^{-/-}$embryos from E12.5 to E13.5 suggest that SDF-1 is not involved in proliferation of PGCs within the gonads; instead, it plays a role in homing of PGCs into the gonads. Down-regulation of cell-surface expression of CXCR4 in E13.5 immotile PGCs compared with CXCR4 expression in E11.5 motile PGCs supports the idea. Further studies will be needed to determine the involvement of SDF-1 in emigration, proper attachment, and/or survival of PGCs during the process of colonization. Furthermore, it is important to note that because the phenotype of SDF-1 $1^{-/-}$embryos in PGC development was not absolute, there might be partial compensation by other chemokines or cytokines. In SDF-1 $1^{-1-}$ mice, homing of PGCs into genital ridges was impaired, but emigration of PGCs into the endoderm or mesentery of hindgut was not affected. This suggests that there may be parallels between SDF-1 functions in hematopoiesis and PGC development because the requirement for SDF-1 in myelopoiesis might be specific to the movement from fetal liver to bone marrow (11, 15-18). Cytokines including BMP-4, BMP-8b, and stem cell factor have been shown to be essential for PGC development. In addition, mice deficient for gp130, a component of receptor complexes for several cytokines including LIF and IL-6, have reduced numbers of PGCs in E11.5 or E12.5 gonads (27). However, the roles for these cytokines in colonization of the gonads by PGCs remain elusive. Transforming growth factor- $\beta$ has been shown to have a chemotactic effect on murine PGCs in vitro (28), but little is known about the physiological roles of transforming growth factor- $\beta$ in PGC mobilization. Thus, SDF-1 is the first cytokine that is involved in homing of PGCs into the gonads during ontogeny in the mouse. In addition, our results reveal that signaling through $G$ protein-coupled receptors, as well as serine/threonine kinase receptors and receptor tyrosine kinases, is required for PGC development in the mouse. Chemokines are known to activate integrin functions in hematopoietic cells (29). Because it has been shown that $\beta 1$ integrins are essential for PGCs to colonize the gonad efficiently (30), $\beta 1$ integrins are candidates for the target of SDF-1 functions in PGC development. More recently, it has been reported that most of the zebrafish PGCs are randomly scattered in the mutants in which expression of SDF-1 or CXCR4 is blocked, showing that SDF-1 is essential for directional migration of PGCs in zebrafish $(31,32)$. Because 
mechanisms of PGC development differ in different animals, it is interesting and important from an evolutionary standpoint to compare the role of SDF-1 between PGC development in fish and mammals. Our results indicate that SDF-1 is involved in mobilization of PGCs in mammals as well as fish. However, considering that PGCs do not lose directionality in their migration along the tissues of embryos in the SDF-1 ${ }^{-1-}$ mice in contrast with the mutant fish, the requirement for SDF-1 in PGC development is likely to be more restricted to the emigration into the gonads in mammals. Further studies will be needed to identify the cytokines or chemokines that compen-

1. Wylie, C. (1999) Cell 96, 165-174.

2. Lawson, K. A., Dunn, N. R., Roelen, B. A., Zeinstra, L. M., Davis, A. M. Wright, C. V., Korving, J. P. \& Hogan, B. L. (1999) Genes Dev. 13, 424-436.

3. Ying, Y., Liu, X. M., Marble, A., Lawson, K. A. \& Zhao, G. Q. (2000) Mol. Endocrinol. 14, 1053-1063.

4. Mintz, B. \& Russell, E. S. (1957) J. Exp. Zool. 134, 207-237.

5. Godin, I., Deed, R., Cooke, J., Zsebo, K., Dexter, M. \& Wylie, C. C. (1991) Nature 352, 807-809.

6. Dolci, S., Williams, D. E., Ernst, M. K., Resnick, J. L., Brannan, C. I., Lock, L. F., Lyman, S. D., Boswell, H. S. \& Donovan, P. J. (1991) Nature 352, 809-811.

7. Matsui, Y., Toksoz, D., Nishikawa, S., Nishikawa, S., Williams, D., Zsebo, K. \& Hogan, B. L. (1991) Nature 353, 750-752.

8. Buehr, M., McLaren, A., Bartley, A. \& Darling, S. (1993) Dev. Dyn. 198, 182-189.

9. Baggiolini, M., Dewald, B. \& Moser, B. (1997) Annu. Rev. Immunol. 15, 675-705.

10. Nagasawa, T., Kikutani, H. \& Kishimoto, T. (1994) Proc. Natl. Acad. Sci. USA 91, 2305-2309.

11. Nagasawa, T., Tachibana, K. \& Kawabata, K. (1999) Adv. Immunol. 71, 211-228.

12. Bleul, C. C., Farzan, M., Choe, H., Parolin, C., Clark-Lewis, I., Sodroski, J. \& Springer, T. A. (1996) Nature 382, 829-833.

13. Oberlin, E., Amara, A., Bachelerie, F., Bessia, C., Virelizier, J. L., ArenzanaSeisdedos, F., Schwartz, O., Heard, J. M., Clark-Lewis, I., Legler, D. F., et al. (1996) Nature 382, 833-835.

14. Nagasawa, T., Nakajima, T., Tachibana, K., Iizasa, H., Bleul, C. C., Yoshie, O., Matsushima, K., Yoshida, N., Springer, T. A. \& Kishimoto, T. (1996) Proc. Natl. Acad. Sci. USA 93, 14726-14729.

15. Nagasawa, T., Nakajima, T., Tachibana, K., Iizasa, H., Bleul, C. C., Yoshie, O., Matsushima, K., Yoshida, N., Springer, T. A. \& Kishimoto, T. (1996) Nature 382, 635-638.

16. Tachibana, K., Hirota, S., Iizasa, H., Yoshida, H., Kawabata, K., Kataoka, Y., Kitamura, Y., Matsushima, K., Yoshida, N., Nishikawa, S., et al. (1998) Nature 393, 591-594. sate the defects in SDF-1 $1^{-/-}$mice. This paper has provided an invaluable window into the molecular mechanisms that control PGC development.

We thank Dr. T. Muramatsu (Nagoya University) for anti-4C9 antibody; M. Yamamoto and D. Okamura for technical advice; and J. Yoshida, K. Matsumoto, and E. Yoshimura for technical assistance. This work was supported by Grants-in-Aid for Scientific Research from the Ministry of Education, Science, Sports, and Culture of Japan and by the Future Program grant of the Japanese Society for the Promotion of Science, Sports, and Culture.

17. Zou, Y. R., Kottmann, A. H., Kuroda, M., Taniuchi, I. \& Littman, D. R. (1998) Nature 393, 595-599.

18. Ma, Q., Jones, D., Borghesani, P. R., Segal, R. A., Nagasawa, T., Kishimoto, T., Bronson, R. T. \& Springer, T. A. (1998) Proc. Natl. Acad. Sci. USA 95, 9448-9453.

19. Feng, Y., Broder, C. C., Kennedy, P. E. \& Berger, E. D. (1996) Science 272, 872-877.

20. Egawa, T., Kawabata, K., Kawamoto, H., Amada, K., Okamoto, R., Fujii, N., Kishimoto, T., Katsura, Y. \& Nagasawa, T. (2001) Immunity 15, 323-334.

21. Aiuti, A., Webb, I. J., Bleul, C., Springer, T. \& Gutierrez-Ramos, J. C. (1997) J. Exp. Med. 185, 111-120.

22. Peled, A., Petit, I., Kollet, O., Magid, M., Ponomaryov, T., Byk, T., Nagler, A. Ben-Hur, H., Many, A., Shultz, L., et al. (1999) Science 283, 845-848.

23. Yoshimizu, T., Sugiyama, N., De Felice, M., Yeom, Y. I., Ohbo, K., Masuko, K., Obinata, M., Abe, K., Scholer, H. R. \& Matsui, Y. (1999) Dev. Growth Differ. 41, 675-684.

24. Yoshinaga, K., Muramatsu, H. \& Muramatsu, T. (1991) Differentiation 48 , 75-82.

25. Ginsburg, M., Snow, M. H. \& McLaren, A. (1990) Development (Cambridge, U.K.) 110, 521-528.

26. Hahnel, A. C., Rappolee, D. A., Millan, J. L., Manes, T., Ziomek, C. A., Theodosiou, N. G., Werb, Z., Pedersen, R. A. \& Schultz, G. A. (1990) Development (Cambridge, U.K.) 110, 555-564.

27. Koshimizu, U., Taga, T., Watanabe, M., Saito, M., Shirayoshi, Y., Kishimoto, T. \& Nakatsuji, N. (1996) Development (Cambridge, U.K.) 88, 1235-1242.

28. Godin, I. \& Wylie, C. (1991) Development (Cambridge, U.K.) 113, 1451-1457.

29. Tanaka, Y., Adams, D. H., Hubscher, S., Hirano, H., Siebenlist, U. \& Shaw, S (1993) Nature 361, 79-82.

30. Anderson, R., Fassler, R., Georges-Labouesse, E., Hynes, R. O., Bader, B. L., Kreidberg, J. A., Schaible, K., Heasman, J. \& Wylie, C. (1999) Development (Cambridge, U.K.) 126, 1655-1664

31. Doitsidou, M., Reichman-Fried, M., Stebler, J., Köprunner, M., Dörries, J., Meyer, D., Esguerra, C. V., Leung, T. \& Raz, E. (2002) Cell 111, 647-659.

32. Knaut, H., Werz, C., Geisler, R., The Tübingen 2000 Screen Consortium \& Nüsslein-Volhard, C. (2003) Nature 421, 279-282. 